\title{
A RELAXATION MODEL TO DESCRIBE THE SPACE CHARGE COMPENSATION PROCESS FOR PULSED ION BEAMS REPRODUCING RGI-SPECTROMETER MEASUREMENTS
}

\author{
A. Jakob, J. Pozimski \\ Johann-Wolfgang-Goethe-University \\ Robert-Mayer-Straße 2-4, 60053 Frankfurt, Germany
}

\begin{abstract}
In magnetic low energy beam transport (LEBT) sections space charge compensation facilitates the transport of high current beams and reduces emittance growth due to space charge forces. For the transport of pulsed beams, the knowledge of the time necessary to establish space charge compensation (rise time) using the process of residual gas ionisation for the production of compensation particles, is important.

To investigate the rise time of space charge compensation time resolved measurements of the beam potential have been performed using a residual gas ion energy analyser (RGIEA). Comparisons between the measured data and the simple model of constant particle capture showed significant deviations. To reproduce the measurements a simplified mathematical description of the temporal potential development was developed and will be presented. It can be used for on-line determination of several factors affecting the rise time and the compensation degree. The calculations of the time dependent potentials will be compared with measurements.
\end{abstract}

\section{INTRODUCTION}

Magnetic LEBT systems enable space charge compensated transport of high perveance ion beams by accumulation of charged particles within the beam potential. Due to the interaction of beam ions and residual gas atoms, electrons and residual gas ions are produced. For positively charged beam ions the electrons are accumulated [1].

For pulsed beams the beam potential is not time constant until the production and loss processes have reached equilibrium. Therefore, in contrary to electrostatic focussing systems and uncompensated transport, a magnetic LEBT system suffers from time dependent space charge forces even for flat top beam pulses. To study the compensation process different diagnostic tools have been developed $[2,3,4]$.

Therefore the residual gas ions (RGI) produced and expelled radially by the self field of the beam can be used. Under assumption of neglectable start energy, the kinetic energy of the RGI corresponds to the beam potential at the production point. The energy distribution of the RGI contains information on the radial beam potential distribution and therefrom the degree of compensation.
Measurements of the temporal beam potential development show a characteristic behaviour even for different beam ions $\left(\mathrm{He}^{+}, \mathrm{H}^{+}, \mathrm{Ar}^{+}\right)$. The measurements proof that the development of the beam potential can not be described by the assumption of electron accumulation solely. In particular the slow decrease of the potential at the beginning of the compensation process (fig. 2) is astonishing. To reproduce the measured potential curves a simple mathematical model was developed.

\section{EXPERIMENTAL SETUP}

The LEBT section used for the investigations consists of a volume source (i.g. $10 \mathrm{keV}$, repetition rate $1 \mathrm{kHz}$, duty cycle $90 \%$ ), two solenoids $\left(\mathrm{B}_{\max } \sim 0.8 \mathrm{~T}\right)$ and a diagnostic section including a RGIEA [5] equipped with a single particle detector (channeltron, $\Delta \mathrm{t}_{\min }=125 \mathrm{~ns}$ ).

\section{THEORY}

Three different interacting processes influencing the temporal development of the beam potential are identified. To simplify the mathematical description an exponential expression was chosen for each of the below listed processes.

(1) $\varphi(t)=\varphi\left(t_{0}\right) \cdot \exp \left[-\frac{t}{\tau}\right]$

I) The reduction of the net charge density (NCD) by accumulation of electrons yields a decreasing potential between the beam edge (BE) and beam axis (BA). Using expression (1) the potential depression inside the beam is:

$$
\text { (1a) } \Delta \Phi_{\mathrm{IB}}(\mathrm{t})=\Phi_{\mathrm{BA}}(\mathrm{t})-\Phi_{\mathrm{BE}}(\mathrm{t})=\underbrace{\frac{\mathrm{I}_{\mathrm{BI}}}{4 \cdot \pi \cdot \varepsilon_{0} \cdot \mathrm{v}_{\mathrm{BI}}}}_{\Delta \Phi_{\mathrm{IB}}\left(\mathrm{t}_{0}\right)} \cdot \exp \left(-\frac{\mathrm{t}}{\tau_{\mathrm{NCD}}}\right)
$$

with the relaxation time $\tau_{\mathrm{NCD}}$ given by the production rate, the beam current $\mathrm{I}_{\mathrm{BI}}$ and the beam ion velocity $\mathrm{v}_{\mathrm{BI}}$. This is the usual approach to describe the temporal development of the beam potential in the model of constant particle capture summarizing all loss processes in the exponential term.

II) The permanent replacement of the thermalized compensation electrons due to the production and loss mechanisms yields a concentration of electrons on the beam axis ("cooling") and therefrom a further decrease of the beam axis potential. Assuming (for simpilcity) a cylindrical and homogenious electron cloud of radius $\mathrm{R}_{\mathrm{e}}(\mathrm{t})$ and using (1) it follows: 
(2) $R_{\mathrm{e}}(t)=\Delta R_{e} \cdot \exp \left(-\frac{t}{\tau_{e}}\right)+R_{e, \text { comp }}$

where $\Delta \mathrm{R}_{\mathrm{e}}$ is the radius shift and $\mathrm{R}_{\mathrm{e}, \text { comp }}$ is the equilibrium radius.

III) The reduction of the space charge forces during compensation causes a concentration of beam ions on the beam axis. Therefrom an increase of the uncompensated beam potential and a moderation of the reduction of the compensated beam potential is expected. Equivalent to (2) the shrink of the ion beam radius $\mathrm{R}_{\mathrm{BI}}(\mathrm{t})$ is then given by:

(3) $R_{\mathrm{BI}}(t)=\Delta R_{B I} \cdot \exp \left(-\frac{t}{\tau_{B I}}\right)+R_{B I, c o m p}$.

with the radius shift $\Delta \mathrm{R}_{\mathrm{BI}}$ (correlated with the generalized beam perveance), the relaxation time $\tau_{\mathrm{BI}}$ and the radius of the ion beam in the equilibrium state $\mathrm{R}_{\mathrm{BI}, \mathrm{comp}}$. Under assumption of a homogeneous density distribution of the beam ions the time development of the beam potential at the position $r$ is given by:

(4)

$\Phi_{\text {BI }}(\mathrm{t})=\Delta \Phi_{\text {BI }}\left(\mathrm{t}_{0}\right) \cdot\left[\left(1-\left(\frac{\mathrm{r}}{\mathrm{R}_{\mathrm{BI}}(\mathrm{t})}\right)^{2}\right)+\left(2 \cdot \ln \left(\frac{\mathrm{r}_{\text {wall }}}{\left.\mathrm{R}_{\mathrm{BI}} \mathrm{t}\right)}\right)\right)\right]$

A similar term was used for the electron cloud.

Under the assumption of equal diameters of the ion beam and the electron cloud in the compensated equilibrium state, the time development of the normalized beam axis potential $\phi_{\mathrm{n}}$ (dimensionless!) is given by (5), the beam potential $\Phi_{\mathrm{BI}}\left(\mathrm{t}_{0}\right)$ follows (6).

(5)

$$
\phi_{\mathrm{n}}(t)=\exp \left(-\frac{t}{\tau_{N C D}}\right) \cdot\left[\begin{array}{l}
\ln \left(\Delta R_{B I}+R_{\text {comp }} \cdot \exp \left(-\frac{t}{\tau_{B I}}\right)\right) \\
-\ln \left(\Delta R_{e}+R_{\text {comp }} \cdot \exp \left(-\frac{t}{\tau_{e}}\right)\right)
\end{array}\right]
$$

(6) $\Phi_{\mathrm{BA}}(\mathrm{t})=2 \cdot \Delta \Phi_{\mathrm{IB}}\left(\mathrm{t}_{0}\right) \cdot \phi_{\mathrm{n}}(\mathrm{t})$

To simplify the mathematical term gained by superposition of all three effects a moderation term $\mathrm{D}(\mathrm{t})$ can be used:
(7) $\phi_{\mathrm{n}, \mathrm{D}}(t)=\exp \left(-\frac{t}{\tau} \cdot D(t)\right)$
$D(t)=\left(\frac{t}{\tau}\right)$

Formula (7) summarizes the influence of the relaxation time for the total system in one free parameter $(\tau)$ given by the individual relaxation times $\left(\tau_{\mathrm{BI}}, \tau_{\mathrm{e}}\right.$ and $\left.\tau_{\mathrm{NCD}}\right)$ for each process. The comparision between the results gained from the much simpler expression (7) and formula (5) is shown in fig. 1 and proves a sufficient agreement between both. Additionally the maximum compensation degree (CD) reached in the experiments (up to $90 \%$ ) has to be taken into account: (8).

$\phi_{\mathrm{n}, \mathrm{D}, \mathrm{CD}}(\mathrm{t})=\mathrm{CD} \cdot \exp \left(-\left(\frac{\mathrm{t}}{\tau}\right)^{2}\right)+(1-\mathrm{CD})$
Using formula (8) and variing $\tau$ and $C D$ a very good reproduction of the measured data can be reached.

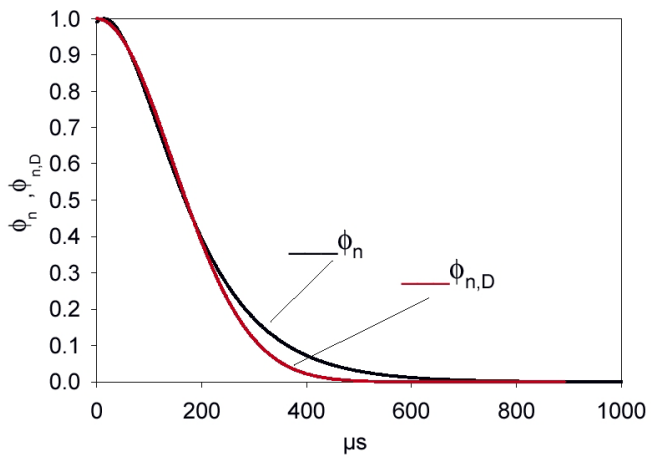

Fig.1: Comparison of expr. (5) \& (7): $\tau=205 \mu \mathrm{s}$, $\mathrm{R}_{\mathrm{BI}, \mathrm{e}, \mathrm{comp}}=5 \mathrm{~mm}, \quad \Delta \mathrm{R}_{\mathrm{BI}}=15 \mathrm{~mm}, \quad \Delta \mathrm{R}_{\mathrm{e}}=45 \mathrm{~mm}, \quad \tau_{\mathrm{BI}}=60 \mu \mathrm{s}$, $\tau_{\mathrm{e}}=360 \mu \mathrm{s}, \tau_{\mathrm{NCD}}=60 \mu \mathrm{s}$.

\section{THEORY \& MEASUREMENTS}

At the beginning of our investigations the ion source was operated in DC mode and the beam was decompensated by an pulsed external electric field (ring electrode). The temporal development of the beam axis and beam edge potential gained from RGIEA-measurements and the corresponding mathematical fit using expression (8). is shown in fig. $2 \& 3$. The measurements have been performed for different residual gas pressures using an $2.2 \mathrm{~mA} \mathrm{He}{ }^{+}$beam at $10 \mathrm{keV}$.

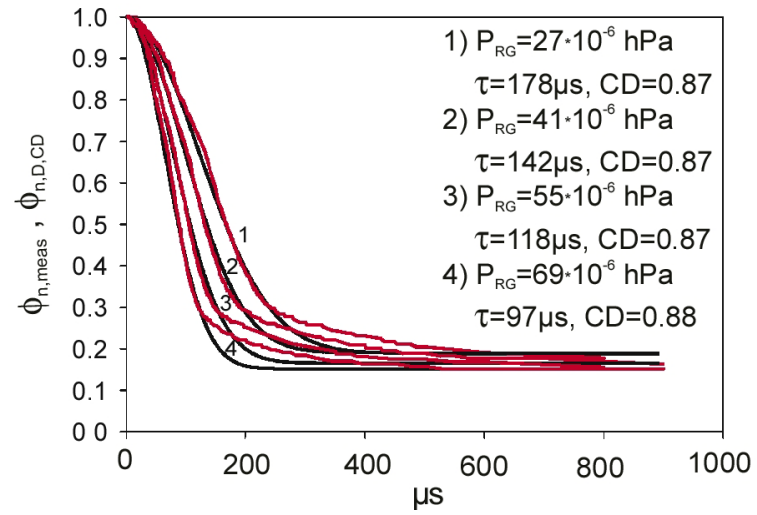

Fig.2 Comparison between $\Phi_{\mathrm{BA}}$-measurements and mathematical fit using expression (8).

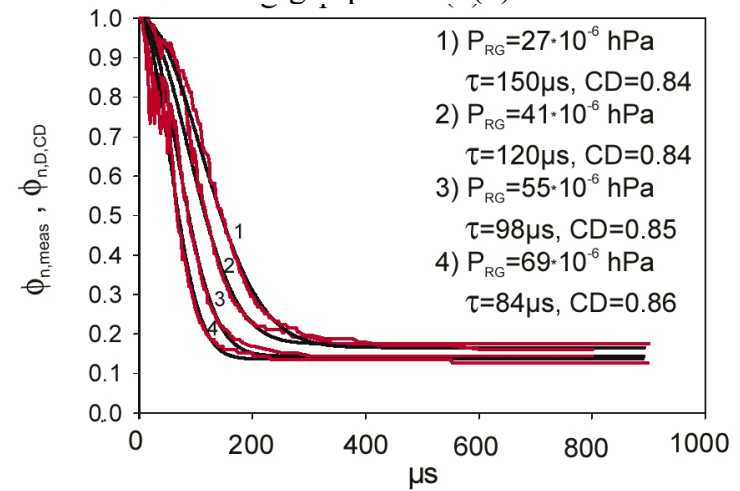

Fig.3: Comparison between $\Phi_{\mathrm{BE}^{-}}$-measurements and mathematical fit using expression (8). 
In a second step the measurements were performed using the ion source in pulsed mode. Therefore the temporal development of the current has to be taken into account. For mathematical fit of the measured currents an exponential term was inserted in expression (8) with the relaxation time of the beam current $\tau_{\mathrm{BC}}$ (see fig. 4).

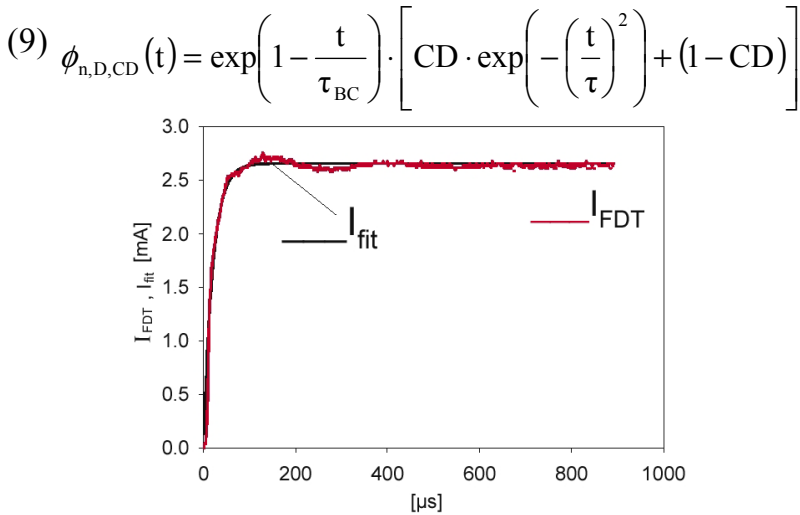

Fig. 4: Comparison of the measured ion beam current $\left(\mathrm{I}_{\mathrm{FDT}}\right)$ and the mathematical fit $\mathrm{I}_{\text {fit }}\left(\tau_{\mathrm{BC}}=20 \mu \mathrm{s}\right)$.

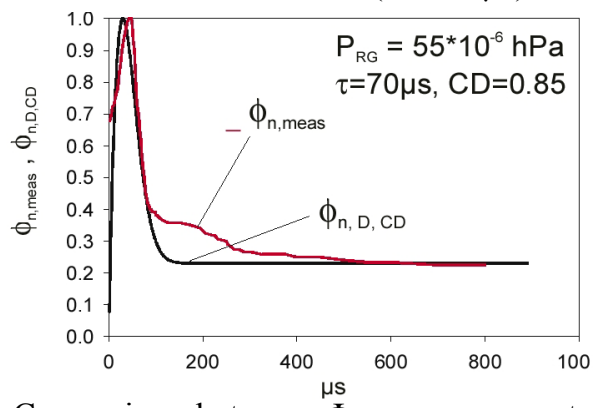

Fig. 5: Comparison between $\Phi_{\mathrm{BA}}$-measurements using a pulsed $10 \mathrm{keV}, 2.7 \mathrm{~mA} \mathrm{He}{ }^{+}$-beam and the mathematical fit.

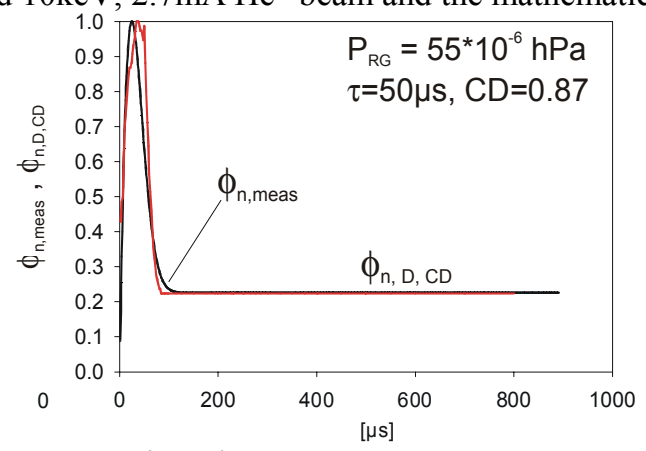

Fig. 6: Comparison between $\Phi_{\mathrm{BE}}$-measurements using a pulsed $10 \mathrm{keV}, 2.7 \mathrm{~mA} \mathrm{He}{ }^{+}$beam and the mathematical fit.

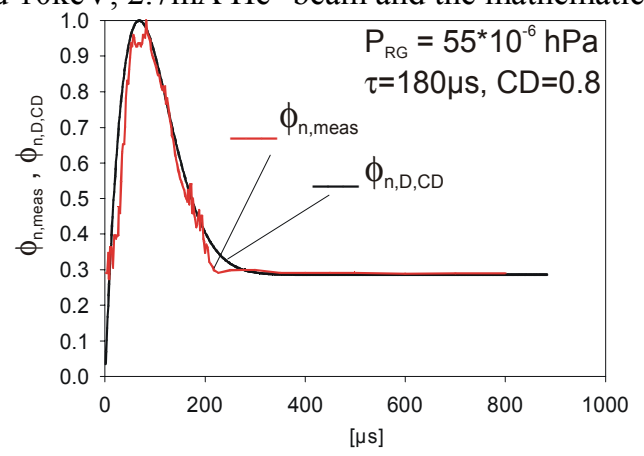

Fig. 7: Comparison between $\Phi_{\mathrm{BA}}$-measurements using a pulsed $10 \mathrm{keV}, 1 \mathrm{~mA} \mathrm{Ar}^{+}$-beam and the mathematical fit using expression (9).

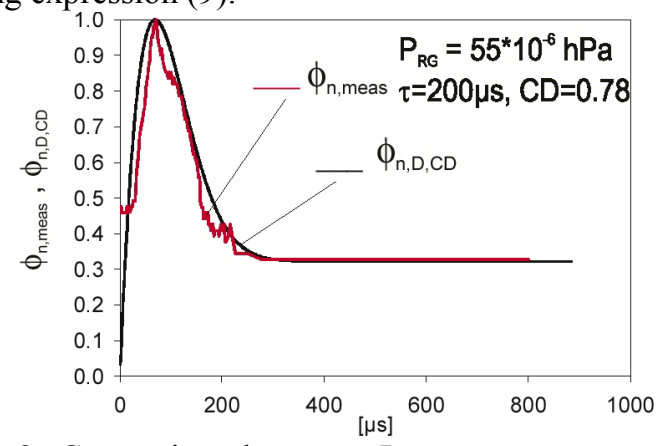

Fig. 8: Comparison between $\Phi_{\mathrm{BE}}$-measurements using a pulsed $10 \mathrm{keV}, 1 \mathrm{~mA} \mathrm{Ar}{ }^{+}$-beam and the mathematical fit.

Fig. $5 \& 6$ show measurements of the beam axis and edge in comparison to the corresponding mathematical fit. The measurements using an $1 \mathrm{~mA} \mathrm{Ar}^{+}$-beam at $10 \mathrm{keV}$, show a similar good correspondence between the measurements and the mathematicall fit as shown in figure 7 and 8 .

\section{SUMMARY}

A simple mathematical description of the temporal beam potential development was found taking three interacting processes (decrease of the NCD, shrinking of the electron cloud and the ion beam) into account. The superposition of the processes together with a variation of the relaxation time $\tau$ and the compensation degree CD allows a very good reproduction of the measured beam potentials. Therefrom a reduction of the total rise time of space charge compensation by influencing the individual processes seems to be possible. Further investigations including the interdependences of the described processes and their influence on the transport properties of the LEBT are planned.

\section{REFERENCES}

[1] A.J.T. Holmes: Theoretical and experimental study of space charge in intense ion beams. Phys. Rev. A 19 (1979) 389

[2] A. Jakob, H. Klein, A. Lakatos, J. Pozimski: Investigation of the Rise of Compensation of High Perveant Ion Beams Using a Time Resolving Ion Energy Spectrometer. Proc. of the EPAC98, p.1538

[3] A. Jakob, H. Klein, A. Lakatos, O. Meusel, J. Pozimski: Time Resolving Diagnostic of the Compensation Process of Pulsed Ion Beams at SILHI. Proc. of the ICIS 99

[4] A. Jakob: Untersuchungen zur zeitaufgelösten Diagnose der Raumladungskompensation intensver Ionenstrahlen. Dissertation, IAP, J.W.G.-Universität Frankfurt, Shaker Verlag, Aachen 2000

[5] A.L. Hughes, V. Rojanski, On the analysis of electronic velocities by electrostatic field, Phys. Rev. 34 (1929) 291 